\title{
Mutations of KRAS/NRAS/BRAF predict cetuximab resistance in metastatic colorectal cancer patients
}

\author{
Hung-Chih Hsu ${ }^{1,2}$, Tan Kien Thiam ${ }^{3}$, Yen-Jung Lu ${ }^{3}$, Chien Yuh Yeh ${ }^{2,4}$, Wen-Sy Tsai ${ }^{2,4}$, \\ Jeng Fu You ${ }^{2,4}$, Hsin Yuan Hung ${ }^{2,4}$, Chi-Neu Tsai ${ }^{5}$, An Hsu ${ }^{3}$, Hua-Chien Chen ${ }^{3}$, \\ Shu-Jen Chen ${ }^{3}$, Tsai-Sheng Yang ${ }^{1,2}$ \\ ${ }^{1}$ Division of Hematology-Oncology, Chang Gung Memorial Hospital, Kwei-Shan, Tao-Yuan 333, Taiwan \\ ${ }^{2}$ College of Medicine, Chang Gung University, Kwei-Shan, Tao-Yuan 333, Taiwan \\ ${ }^{3}$ ACT Genomics, Neihu Dist., Taipei 114, Taiwan \\ ${ }^{4}$ Division of Colon and Rectal Surgery, Chang Gung Memorial Hospital, Kwei-Shan, Tao-Yuan 333, Taiwan \\ ${ }^{5}$ Graduate Institute of Clinical Medicine, College of Medicine, Chang Gung University, Kwei-Shan, Tao-Yuan 333, Taiwan \\ Correspondence to: Shu-Jen Chen, e-mail: sjchen@actgenomics.com \\ Tsai-Sheng Yang, e-mail: a481124@cgmh.org.tw
}

Keywords: BRAF, metastatic colorectal cancer, RAS, mutation, cetuximab resistance

Received: July 17, 2015

Accepted: February 18, 2016

Published: March 12, 2016

\section{ABSTRACT}

Approximately $45 \%$ of metastatic colorectal cancer (mCRC) patients with wild-type KRAS exon 2 are resistant to cetuximab treatment. We set out to identify additional genetic markers that might predict the response to cetuximab treatment. Fifty-three wild-type KRAS exon 2 mCRC patients were treated with cetuximab/ irinotecan-based chemotherapy as a first- or third-line therapy. The mutational statuses of 10 EGFR pathway genes were analyzed in primary tumors using nextgeneration sequencing. BRAF, PIK3CA, KRAS (exons 3 and 4), NRAS, PTEN, and $A K T 1$ mutations were detected in $6,6,5,4,1$, and 1 patient, respectively. Four of the BRAF mutations were non-V600 variants. Four tumors harbored multiple co-existing (complex) mutations. All patients with BRAF mutations or complex mutation patterns were cetuximab non-responders. All patients but one harboring KRAS, NRAS, or BRAF mutations were non-responders. Mutations in any one of these three genes were associated with a poor response rate $(7.1 \%)$ and reduced survival (PFS $=\mathbf{8 . 0}$ months) compared to wild-type patients (74.4\% and 11.6 months). Our data suggest that KRAS, NRAS, and BRAF mutations predict response to cetuximab treatment in mCRC patients.

\section{INTRODUCTION}

Despite recent advances in treatment, nearly 600,000 colorectal cancer- (CRC) related deaths occur annually [1], and CRC is the most common cancer in Taiwanese patients. Many patients with advanced CRC experience recurrence after surgical resection, the primary treatment for this disease. Furthermore, $>25 \%$ of CRC patients have liver metastases at the time of initial diagnosis, and approximately $50 \%$ of patients eventually develop metastases. The 5 -year survival rate is as low as $10 \sim 20 \%$ for patients with distant metastatic disease [2]. While screening, surgery, and medical therapies are effective in the management of early-stage CRC, these treatment options are far less efficacious in advanced stages. Interand intratumoral genetic heterogeneity is key factor in predicting treatment failure and drug resistance in CRC therapies [3-4].

In the past decade, targeted biologic therapies, including monoclonal antibodies targeting vascular endothelial growth factor (VEGF) and epidermal growth factor receptor (EGFR), have significantly improved clinical outcomes in patients with CRC [5]. Ligand-induced EGFR activation in particular plays a pivotal role in tumor proliferation, invasion, migration, and neovascularization through the RAS-RAF-MAPK and PI3K-AKT-mTOR 
pathways [6]. The EGFR-targeting antibodies cetuximab, a chimeric IgG1 monoclonal antibody, and panitumumab, a humanized IgG2 monoclonal antibody, have proven effective against $\mathrm{CRC}$ in clinical trials and have been used either individually or in combination with standard chemotherapy to improve survival in metastatic CRC $(\mathrm{mCRC})$ patients [7-8]. However, mutations in effector signaling molecules downstream of EGFR activate receptor-independent pathway (s) that render tumors unresponsive to EGFR inhibition treatment. Randomized phase III studies provide compelling evidence that the EGFR-targeting monoclonal antibodies cetuximab and panitumumab are effective only in CRC patients harboring wild-type $K R A S$ exon 2 [9-10]. Consequently, regulatory authorities have mandated the implementation of $K R A S$ exon 2 mutation screening when selecting patients for anti-EGFR treatment $[8,11]$.

Although the KRAS exon 2 mutation has been established as an important biomarker for predicting responsiveness to anti-EGFR treatment, approximately $40 \sim 50 \%$ patients harboring wild-type $K R A S$ exon 2 do not benefit from these targeted agents, suggesting the potential involvement of genetic alterations in the KRAS/ BRAF and PI3K/AKT pathways downstream of EGFR. All of these genes have been associated with tumor growth and progression, and recent studies suggest that additional mutations in $K R A S$ and $N R A S$, as well as downstream mutations in $B R A F$ or $P I K 3 C A$, may cause resistance to anti-EGFR treatment [12]. However, these findings were based on clinical studies containing patients of different races in which few genes were analyzed, and the results were often contradictory [13]. Previous studies have found higher rates of $K R A S$ mutations in Asian patients with small-cell lung carcinoma, reflecting an ethnic difference in cancer genomics [14]. However, factors influencing $K R A S$ and other effectors downstream of EGFR in CRC specifically in Asian populations have not been systemically evaluated, particularly in the Taiwanese population. Additional molecular studies identifying other predictive biomarkers may help to optimize anti-EGFR therapies in $\mathrm{mCRC}$ patients.

Since the launch of the first massively parallel sequencing platform in 2005 , next-generation sequencing (NGS) technologies have evolved rapidly, revolutionizing the scale of genomic studies and providing powerful diagnostic tools for implementing precision medicine through high-throughput genomic analysis [15]. As NGS techniques continue to improve and decrease in cost, it has become feasible to routinely employ NGS in clinical settings to analyze large-scale genetic information regarding inter- and intratumoral gene alterations, allowing better stratification of patients when selecting personalized therapies. In the present study, we utilized NGS technology to analyze the EGFR signaling pathway genes EGFR, KRAS, HRAS, NRAS, BRAF, PIK3CA,
AKT1, PTEN, HER2, and HER4 in a wild-type KRAS exon 2 cohort of 53 Taiwanese $\mathrm{mCRC}$ patients undergoing cetuximab treatment. Our results demonstrate that poor responses to cetuximab in CRC patients can be attributed to a combinational set of gene mutations in addition to mutations in $K R A S$ exon 2.

\section{RESULTS}

\section{Patient characteristics}

A total of $53 \mathrm{mCRC}$ patients were treated with cetuximab in combination with chemotherapy either as a first line $(n=39,73.5 \%)$ or third line $(n=14,26.4 \%)$ therapy. All subjects were confirmed as having wild-type $K R A S$ exon 2 before cetiximab administration. Followups were conducted with each patient every week to three months until March 1, 2014, or until death. The mean follow-up duration was 17.1 months, with a standard deviation of 10.1 months. Patient age at diagnosis ranged from 28 to 93 years (mean, $63.5 \pm 14.0$ ). The tumor subsites were the colon (32 patients) and rectum (21 patients). Tumors were staged according to the AJCC 2010 guidelines. The main characteristics of all enrolled patients are summarized in Table 1.

The cohort was separated into two groups based on patients' responses to cetuximab. The "responder" group included $30(61.5 \%)$ patients with either complete remission $(n=2,3.8 \%)$ or partial remission $(n=28$, $52.8 \%$ ). The "non-responder" group included 23 (43.4\%) patients with stable disease $(n=18,34 \%)$ or progression $(n=5,9.4 \%)$. Response rates were similar among the firstline subjects $(61.5 \%, 24$ of 39$)$ and the third-line subjects $(42.9 \%, 6$ of 14$)(p=0.346)$. Median response durations were also similar among first-line subjects (10.83 months) and third-line subjects (11.6 months) $(p=0.370)$.

\section{Targeted sequencing of genes involved in EGFR signaling pathway}

In order to identify genetic alterations in the EGFR signaling pathway that affect cetuximab treatment responses, we applied NGS technology to analyze frequently mutated regions of 10 EGFR-related genes, including EGFR, KRAS, HRAS, NRAS, BRAF, PIK3CA, $A K T 1, P T E N, H E R 2$, and HER4 (targeted regions listed in Supplementary Table S1). The TP53 gene, one of the most frequently mutated genes in CRC, was sequenced as a control. All samples were sequenced at an average depth of $>1000 \times($ Supplementary Table S2). Raw sequence data were aligned to a human reference genome to identify variants. After annotation, variants were filtered to remove single nucleotide polymorphisms (SNPs) and synonymous mutations. Only the non-synonymous mutations were used for subsequent analysis. 
Table 1: Characteristics of patients with metastatic colorectal cancer

\begin{tabular}{|c|c|c|c|c|}
\hline & All patients & Responders & Non-responders & $p$-value* \\
\hline & $N(\%)$ & $N(\%)$ & $N(\%)$ & \\
\hline Total number & $53(100)$ & $30(56.6)$ & $23(43.4)$ & \\
\hline Sex & & & & 0.775 \\
\hline Male & $34(64.2)$ & $20(66.7)$ & $14(60.8)$ & \\
\hline Female & $19(35.8)$ & $10(33.3)$ & $9(39.2)$ & \\
\hline Age & & & & 0.052 \\
\hline$\leq 70$ & $40(75.5)$ & $26(86.7)$ & $14(60.9)$ & \\
\hline$>70$ & $13(24.5)$ & $4(13.3)$ & $9(39.1)$ & \\
\hline Median (range) & $57(32-83)$ & $53.5(32-83)$ & $61(36-79)$ & \\
\hline Histologic Grade & & & & 0.222 \\
\hline low grade $^{1}$ & $47(60)$ & $28(63.3)$ & $19(56.5)$ & \\
\hline high grade ${ }^{2}$ & $6(40)$ & $2(36.7)$ & $4(43.5)$ & \\
\hline Metastatic pattern & & & & 0.375 \\
\hline metachronous & $17(66)$ & $9(72.9)$ & $8(57.1)$ & \\
\hline synchronous & $36(34)$ & $21(27.1)$ & $15(42.9)$ & \\
\hline Primary Tumor site & & & & 0.778 \\
\hline Colon & $32(60.4)$ & $19(63.3)$ & $13(56.5)$ & \\
\hline Rectum & $21(39.6)$ & $11(36.7)$ & $10(43.5)$ & \\
\hline \multicolumn{5}{|l|}{ Metastatic site } \\
\hline Liver & 38 & 22 & 16 & 0.962 \\
\hline Lung & 19 & 10 & 9 & 0.663 \\
\hline Other & 22 & 8 & 14 & 0.012 \\
\hline $\begin{array}{l}\text { Number of metastatic } \\
\text { site }\end{array}$ & & & & 0.052 \\
\hline 1 & 31 & 21 & 10 & \\
\hline$>1$ & 22 & 9 & 13 & \\
\hline Treatment regimen & & & & 0.346 \\
\hline 1st line & $39(74)$ & $24(80.0)$ & $15(65.2)$ & \\
\hline 3rd line & $14(26)$ & $6(20.0)$ & $8(34.8)$ & \\
\hline PFS (months) & & & & 0.061 \\
\hline Median (range) & $10.6(2.7-51.7)$ & $11.4(4.4-51.7)$ & $8(2.7-26.4)$ & \\
\hline
\end{tabular}

*: Fisher exact $p$-value.

1: well-differentiated/moderately-differentiated.

2: poorly-differentiated.

We detected 61 non-synonymous variants in 7 genes, including $A K T 1, B R A F, K R A S, N R A S, P I K 3 C A, P T E N$, and TP53 (Figure 1, Supplementary Table S3), in 40 subjects. As expected, TP53 was the most frequently mutated gene detected in the cohort. Thirty-three subjects exhibited mutations in the TP53 gene, resulting in a population frequency of $62.3 \%$. The second most frequently altered genes were $B R A F$ and $P I K 3 C A$, with mutations detected in 6 subjects $(11.3 \%)$ for each. The third most frequently mutated gene was $K R A S$, with a $9.4 \%(n=5)$ population frequency, 
followed by NRAS ( $n=4,7.5 \%), \operatorname{PTEN}(n=2,3.7 \%)$ and AKT1 $(n=1,1.9 \%)$. The mutation frequencies of TP53, $B R A F$, and $N R A S$, but not $K R A S$, detected in this study are very similar to those found by Brannon et al. [16-17].

Mutations in EGFR-related genes were detected in $20(38 \%)$ subjects (Figure 1). Sixteen subjects harbored a single mutation while 4 subjects displayed a complex mutation pattern with multiple mutations co-existing in the same tumor. Interestingly, 3 of 6 subjects with $B R A F$ mutations displayed complex mutation patterns $(B R A F+A K T 1, \quad B R A F+P I K 3 C A, B R A F+N R A S)$. Two complex mutation patterns were observed for $P I K 3 C A$ mutations (PIK3CA+BRAF, PIK3CA+KRAS). These results indicate that, while $K R A S$ and $N R A S$ tend to exhibit mutually exclusive mutation patterns, mutations in signaling molecules downstream of EGFR may co-exist with KRAS and NRAS alterations in colorectal tumors.

\section{Genetic alterations are more common in cetuximab non-responders}

We next compared the mutation frequencies of individual genes between cetuximab responders and non-responders. As shown in Figure 2A and Table 2, mutation frequencies of TP53 (63.3\% for responders vs. $60.9 \%$ for non-responders, Fisher's exact test: $p=1.000$ ) and PIK3CA (10\% for responders vs. $13 \%$ for nonresponders, $p=1.000)$ were similar between responders and non-responders. In contrast, $B R A F$ mutations $(0 \%$ for responders vs. $26.1 \%$ for non-responders, $p=0.0044)$ and $K R A S(0 \%$ for responders vs. $21.7 \%$ for non-responders, $p=0.0117)$ were observed exclusively in non-responders. Three of the 4 subjects with NRAS mutations were nonresponders $(p=0.3053)$ while both PTEN mutations were detected in responders $(p=0.4993)$. Collective mutation frequencies of $K R A S, N R A S$, and $B R A F$ combined were higher in non-responders $(p=0.0001)$.
Among the 23 non-responders, 4 (17.4\%) subjects had a non-exon $2 K R A S$ mutation, 3 (13\%) subjects had a $B R A F$ mutation, and 2 subjects $(8.7 \%)$ had an $N R A S$ mutation (Figure 2B). In addition to single mutations, 4 subjects showed complex mutations in both the KRAS/ $P I K 3 C A, B R A F / N R A S, B R A F / A K T 1$, and BRAF/PIK3CA genes. Collectively, genetic alterations in $B R A F, N R A S$ and $K R A S$ were detected in $13(56.5 \%)$ of the nonresponders. These genetic alterations may contribute to resistance to cetuximab; the mechanism of cetuximab resistance for the remaining $10(43.5 \%)$ subjects requires further investigation.

\section{Genetic alterations related to cetuximab resistance}

To further explore the relationship between genetic alterations and cetuximab resistance, we analyzed the $K R A S, N R A S$, and $B R A F$ mutant alleles detected in non-responders and compared mutation patterns of the individual genes to data published by the Cancer Genome Atlas (TCGA) Research Network [17]. Activating mutations in a few well-characterized loci were detected in the $K R A S$ and $N R A S$ genes (Figure 3A, 3B). For example, two Q61L (exon 3), one Q61H (exon 3), and one A146T (exon 4) KRAS mutations were detected in the non-responders. All of these rare mutations activate KRAS activity [18-19]. These loci are not included in the routine $K R A S$ exon 2 test but are frequently mutated in CRC patients. Similarly, activating NRAS mutations (G12A, G13D and Q61K) are not included in routine tests for $\mathrm{CRC}$ patients before cetuximab administration. Mutations in both genes are frequently detected in CRC patients and are present at a higher frequency in cetuximab nonresponders compared with responders. These additional $R A S$ mutations were also verified in large randomized trials and are included in the all-RAS mutational test

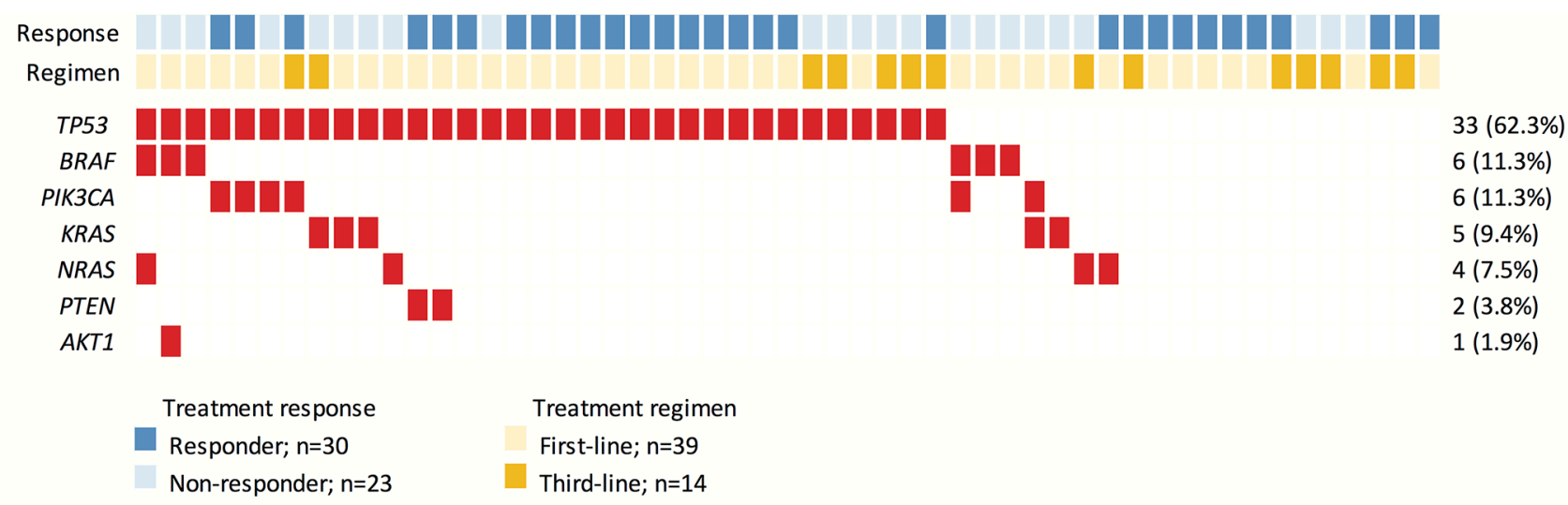

Figure 1: Heat-map representation of individual non-silent variants identified in a total of 53 specimens. Light blue and dark blue highlighting indicates individual responses to cetuximab-based treatment. Light and dark yellow highlighting indicates which cetuximab-based regimen each individual received. Individuals highlighted in red had non-silent mutations. The columns in the Table denote the samples, and the rows denote the genes. The right panel indicates the mutation frequency of each gene in the cohort. 
recently recommended by the National Comprehensive Cancer Network [20].

Among the $B R A F$ mutations observed in CRC, a single nucleotide mutation in codon 600 of the kinase domain resulting in substitution of glutamic acid for valine (V600E) accounted for over 95\%. In fact, the V600E allele was the only somatic mutation detected in the TCGA CRC samples [21]. In comparison, among the 6 subjects with $B R A F$ mutations in this cohort, only $2(33.3 \%)$ had $B R A F$ V600E. Other genetic alterations in $B R A F$ included G466A (1 subject), G469A (2 subjects) and D594G (1 subject) (Figure 3C). These three mutations also occurred in the protein kinase domain and were not recorded in the TCGA colorectal cancer dataset. Furthermore, previous studies suggest that $P I K 3 C A$ exon 20 mutations, but not $P I K 3 C A$ exon 9 mutations, are associated with cetuximab resistance in CRC [22]. PIK3CA mutations in exon 9 (helical domain) were detected in 5 subjects, and only 1 subject had a PIK3CA mutation in exon 20 (kinase domain) (Supplementary Figure S1). The high frequency of PIK3CA exon-9 mutations (E542K and E545K) was similar to that observed in Japanese CRC patients [23]. All PIK3CA mutations identified in this study have been reported in metastatic $\mathrm{CRC}$ before.

\section{Genetic alterations and treatment response}

In the responder group, 2 patients showed complete response (CR) and 28 showed partial response (PR) for a $60.1 \%$ overall response rate (ORR) to cetuximab. In the non-responder group, 18 patients had stable disease (SD) and 5 had progressive disease (PD). The waterfall plot illustrated the relationship between genetic alterations and treatment responses (Figure 4). All patients with mutations in $K R A S$ or $B R A F$ failed to respond to cetuximab treatment, and three subjects with $N R A S$ mutations did not respond to cetuximab treatment. One subject with a low NRAS G12C mutant allele frequency (5\%) did show a $50 \%$ tumor reduction after cetuximab treatment. Overall, tumors harboring mutations in the KRAS-NRAS-BRAF axis responded poorly to cetuximab-based treatment. Responses to cetuximab-based treatment in tumors harboring mutations in the PTEN-PI3K-AKT signaling pathway were more complex. Both tumors harboring PTEN mutations responded well to the treatment. All four subjects with a single PIK3CA mutation showed weak to moderate tumor regression $(-14 \%,-34 \%,-36 \%$, and $-37 \%)$.

In addition to single mutations, complex mutational patterns were frequently detected in mCRC patients. Notably, none of the tumors harboring complex mutations responded to cetuximab-based treatment. Only one tumor harboring complex $B R A F / N R A S$ mutations showed weak tumor regression $(-24 \%)$. The remaining three tumors with complex mutations showed disease progression $(4 \%$, $14 \%$ and $21 \%$ changes in tumor size) during cetuximabbased treatment. These results suggest that these complex mutational patterns also predict poorer response toward cetuximab treatment. We used a microsatellite instability (MSI) test to determine whether complex mutational patterns were associated with genetic hypermutability caused by impaired DNA mismatch repair (MMR). All samples with complex mutations showed the microsatellite stable (MSS) phenotype, suggesting that the concomitant mutations represent a complex perturbation of the RAS/RAF signaling pathway in advanced CRC rather than a consequence of MSI-associated hypermutation. Furthermore, the frequency of individual mutant alleles differed in some of the tumors harboring complex mutational patterns. For example, tumor A00021 had both $A K T 1$ E17K (27.9\%) and BRAF G469A (19.7\%) mutations, and tumor A00026 had both BRAF D594G
A

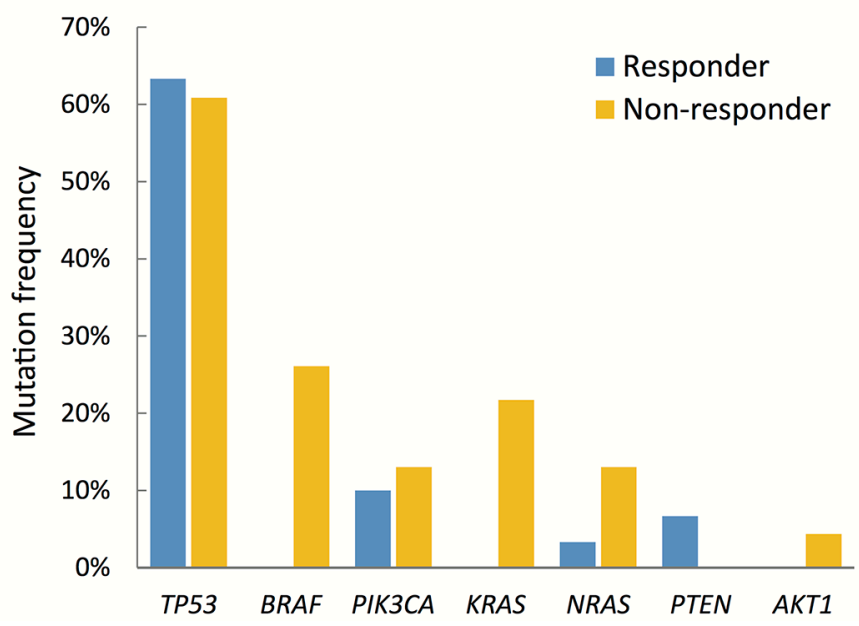

B

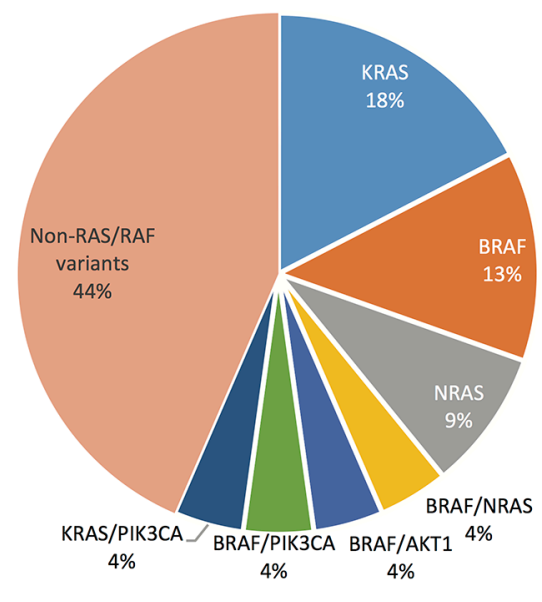

Figure 2: Extent of genetic disruption in metastatic CRC. (A) Prevalence of tumors harboring non-silent mutations in cetruximab responders (blue) and non-responders (yellow). (B) Distribution of genetic disruptions in the cetuximab non-responder group. 
Table 2: Association between genetic alterations and treatment outcome

\begin{tabular}{|c|c|c|c|c|}
\hline \multirow{2}{*}{$\begin{array}{c}\text { Gene } \\
\text { symbol }\end{array}$} & \multirow{2}{*}{$\begin{array}{c}\text { Mutation } \\
\text { status }\end{array}$} & \multicolumn{2}{|c|}{ Treatment outcome } & \multirow{2}{*}{$p$ value* } \\
\hline & & Responders $(n=30)$ & Non-responders $(n=23)$ & \\
\hline \multirow{2}{*}{$B R A F$} & WT & 30 & 17 & \multirow{2}{*}{0.004} \\
\hline & Mut & 0 & 6 & \\
\hline \multirow{2}{*}{$K R A S$} & WT & 30 & 18 & \multirow{2}{*}{0.012} \\
\hline & Mut & 0 & 5 & \\
\hline \multirow{2}{*}{$N R A S$} & WT & 29 & 20 & \multirow{2}{*}{0.305} \\
\hline & Mut & 1 & 3 & \\
\hline \multirow{2}{*}{$\begin{array}{c}B R A F / \\
K R A S / N R A S\end{array}$} & WT & 29 & 10 & \multirow{2}{*}{0.000} \\
\hline & Mut & 1 & 13 & \\
\hline \multirow{2}{*}{$P I K 3 C A$} & WT & 27 & 20 & \multirow{2}{*}{1.000} \\
\hline & Mut & 3 & 3 & \\
\hline \multirow{2}{*}{$A K T 1$} & WT & 30 & 22 & \multirow{2}{*}{0.434} \\
\hline & Mut & 0 & 1 & \\
\hline \multirow{2}{*}{ PTEN } & WT & 28 & 23 & \multirow{2}{*}{0.499} \\
\hline & Mut & 2 & 0 & \\
\hline \multirow{2}{*}{$\begin{array}{c}\text { PIK3CA/ } \\
\text { AKT1/ } \\
\text { PTEN }\end{array}$} & WT & 25 & 19 & \multirow{2}{*}{1.000} \\
\hline & Mut & 5 & 4 & \\
\hline \multirow{2}{*}{ TP53 } & WT & 11 & 9 & \multirow{2}{*}{1.000} \\
\hline & Mut & 19 & 14 & \\
\hline
\end{tabular}

*: Fisher exact $p$-value

(26.7\%) and PIK3CA E542K (15.6\%) mutations. Such differences in mutant allele frequencies implies complex intra-tumor heterogeneity, which may also contribute to the lack of response to cetuximab.

\section{Genetic alteration and duration of treatment response}

In addition to changes in tumor size, we also examined whether genetic mutations affected the duration of response. The median duration of response to cetuximab-based treatment was 10.6 months (range, 2.7 to 51.7 months; mean, 11.4 months). The median response durations the 30 responders and the 23 non-responders were 11.4 months (range, 4.4 - 51.7 months; mean, 13.1 months) and 8 months (range, 2.7 to 26.4 months; mean, 9.1 months), respectively. A Kaplan-Meier plot revealed that patients with mutated KRAS exon 3/4 had shorter median response durations than patients with wild-type $K R A S$ (6.3 vs. 11.2 months, respectively; logrank $p$-value $<0.0001$; Figure 5A). Similarly, patients with mutated $B R A F$ had shorter median response durations than patients with wild-type $B R A F$ (7.4 vs. 11.5 months, respectively; log-rank $p$-value $=0.0015$; Figure 5B). Furthermore, patients with mutated KRAS/NRAS/BRAF had lower response rates and shorter median response durations than wild-type patients $(7.1 \%$ vs. $74.4 \%$ and 11.6 vs. 8.0 months, respectively; log-rank $p$-value $=0.0078$; Figure 5C). In contrast, no difference in response duration was observed between patients with mutated and wildtype PIK3CA genes ( $p=0.624$; Figure $5 \mathrm{D})$.

We further evaluated the ability of genetic mutations and other clinicopathological risk factors, including sex (male vs female), age ( $>70$ vs $\leq 70$ ), pT stage (T4 vs $\mathrm{T} 2-3$ ), pN stage (N2 vs N0-1), p-Stage (IV vs I-III), histologic grade (high vs low), metastatic pattern (metachronous vs synchronous), primary tumor site (rectum vs colon), number of metastatic sites $(>1$ vs 1 ), and treatment regimen (3rd-line vs 1 st-line), to predict cetuximab response duration. Univariate analysis revealed that higher age, multiple metastatic sites, $K R A S$ mutations, BRAF mutations, and mutated KRAS/NRAS/ $B R A F$ were associated with shorter response durations (Table 3). Multivariate analysis indicated that the presence of multiple metastatic sites and mutated $K R A S, B R A F$, and $K R A S / N R A S / B R A F$ were independent risk factors for 
A

KRAS

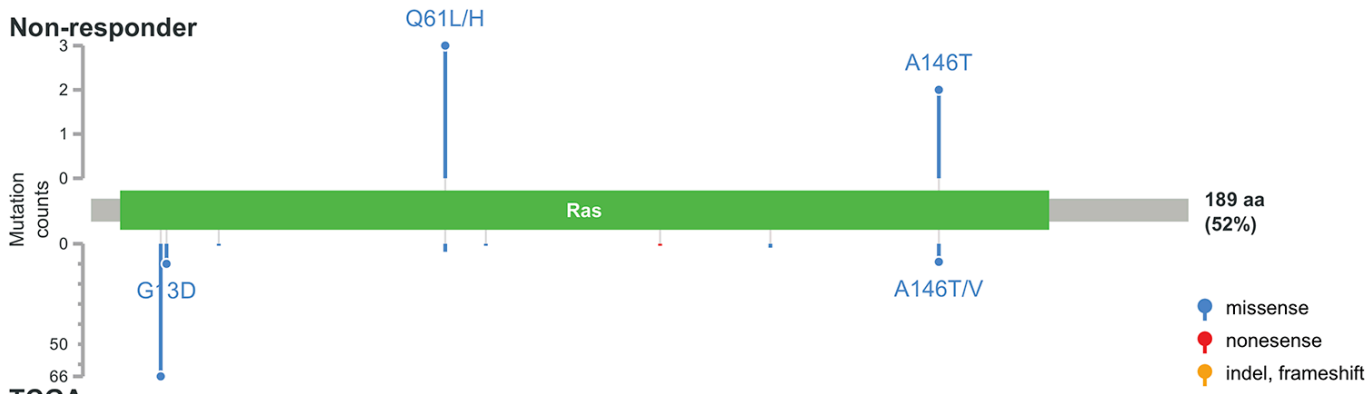

TCGA G12D/N/C/S/AF/R

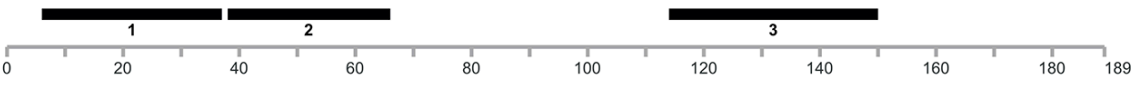

Ras family

B NRAS

Non-responder

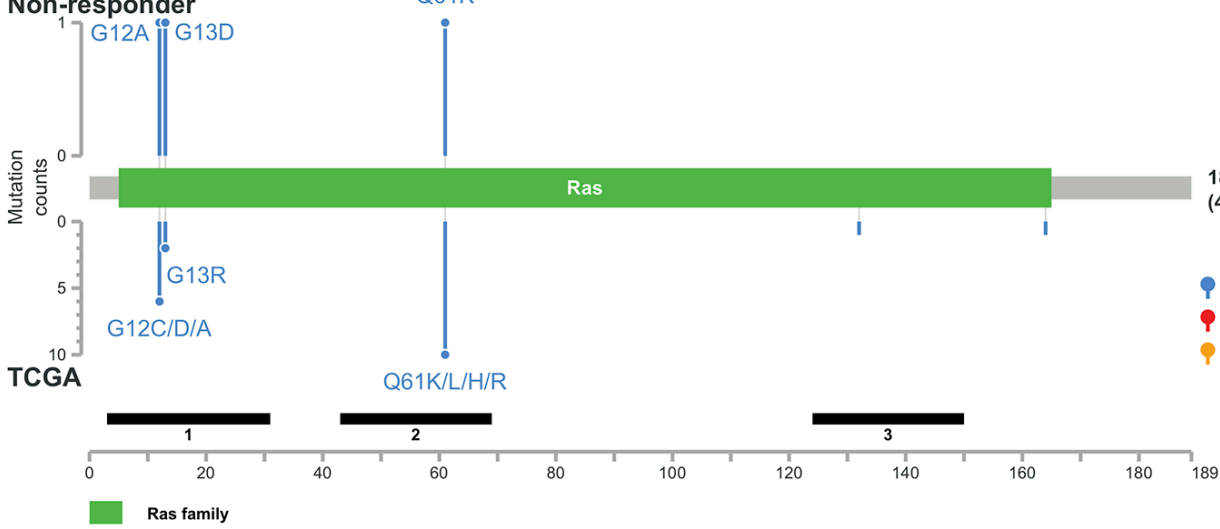

189 aa

(44\%)

missense

Ponesense

indel, frameshift

C

BRAF

Non-responder

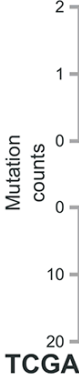
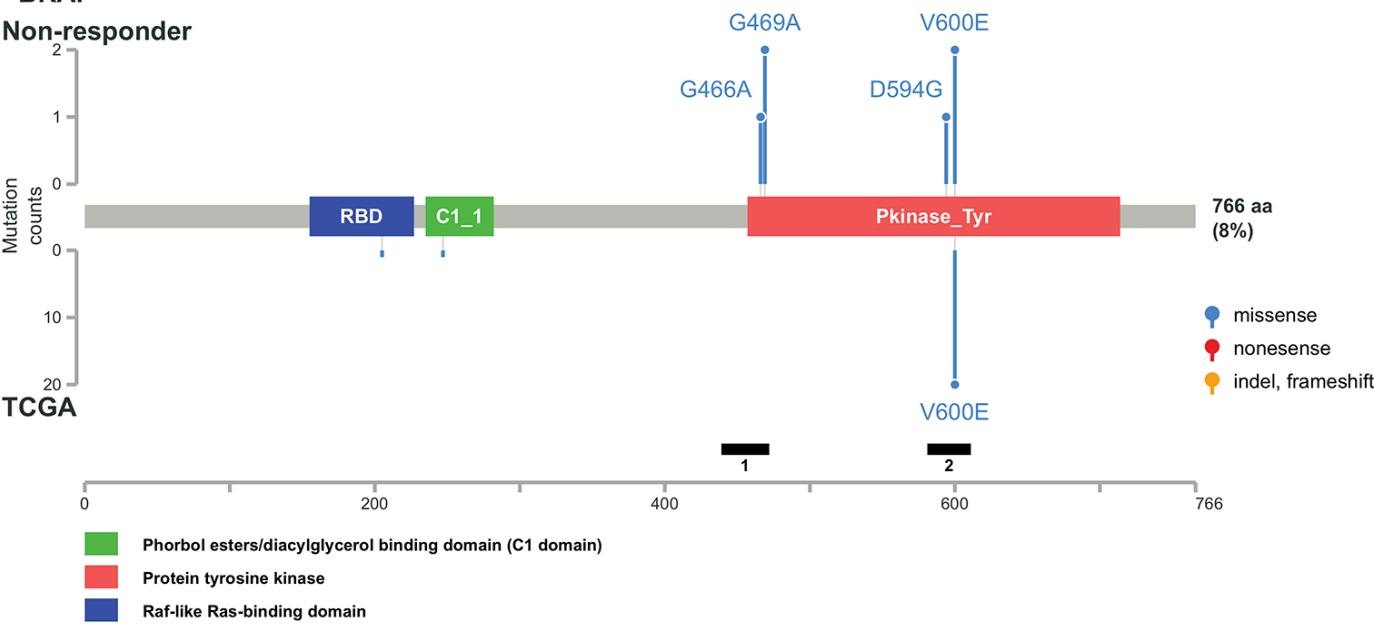

Figure 3: Distribution of genetic alterations detected in the non-responder group. Schematic representation of protein structures of KRAS (A) NRAS (B) and BRAF (C). The upper panel indicates the genetic alterations detected in the non-responder group. The lower panel indicates the mutation spectrum in The Cancer Genome Atlas (TCGA) colorectal cancer dataset. The left scale indicates the number of cases. The blue, yellow, red, and purple circles indicate missense mutations, insertions/deletions, nonsense mutations, and multiple types of mutations, respectively. 
poorer response (Table 3). Hazard ratios for patients with mutated $K R A S, B R A F$, or $K R A S / N R A S / B R A F$, compared to patients with wild-type genes, were $8.479(95 \%$ $\mathrm{CI}=2.524-28.487, p=0.001), 2.603(95 \% \mathrm{CI}=1.017$ 6.661, $p=0.046)$, and $2.716(95 \% \mathrm{CI}=1.345-5.481$, $p=0.005)$, respectively. These results further support that mutated KRAS, BRAF, and KRAS/NRAS/BRAF are independently associated with cetuximab response even after considering traditional risk factors.

\section{DISCUSSION}

To date, KRAS exon 2 mutations are the most commonly used biomarker for predicting the therapeutic efficacy of anti-EGFR antibodies in advanced CRC. However, it has become clear that the KRAS exon 2 mutation alone is insufficient for predicting responsiveness to such therapies. The prognostic and predictive relevance of other genes in the RAS-RAF and PI3K-AKT-mTOR pathways are now being investigated in CRC patients with different ethnic backgrounds. However, the role of these genes is somewhat controversial, in part because previous studies include patients of various races, analyze only a few biomarkers, and employ diverse experimental methods. Here, we analyzed genes in the EGFR signaling pathway in a well-characterized Taiwanese cohort to complete the first study in Asian CRC patients undergoing first- or third-line cetuximab treatment in combination with chemotherapy. Our study clearly illustrates the predictive importance of mutations outside of the KRAS exon 2. Mutations in KRAS exons 3 and 4, $B R A F$, and $N R A S$ served as strong predictors of PFS poor responses to EGFR-targeted therapies. More importantly, our data demonstrate that significantly higher predictive power might be achieved by analyzing combinations of mutations in these 3 genes, which occur in $80 \%$ of cetuximab non-responders.

In the past, multiplex mutation screening was challenging due to inadequate sample availability, poor tumor purity, difficulty in including rarer mutations, and technical obstacles. We overcame these technical problems and established a multiplex mutation assay for clinical CRC samples by exploiting two-step tagging PCR and NGS technology for targeted amplicon sequencing, allowing simultaneous detection of very low levels of point mutations in targeted genes. PCR-based enrichment coupled with the NGS procedure enabled us to perform high-sensitivity mutation profiling in tumor biopsy

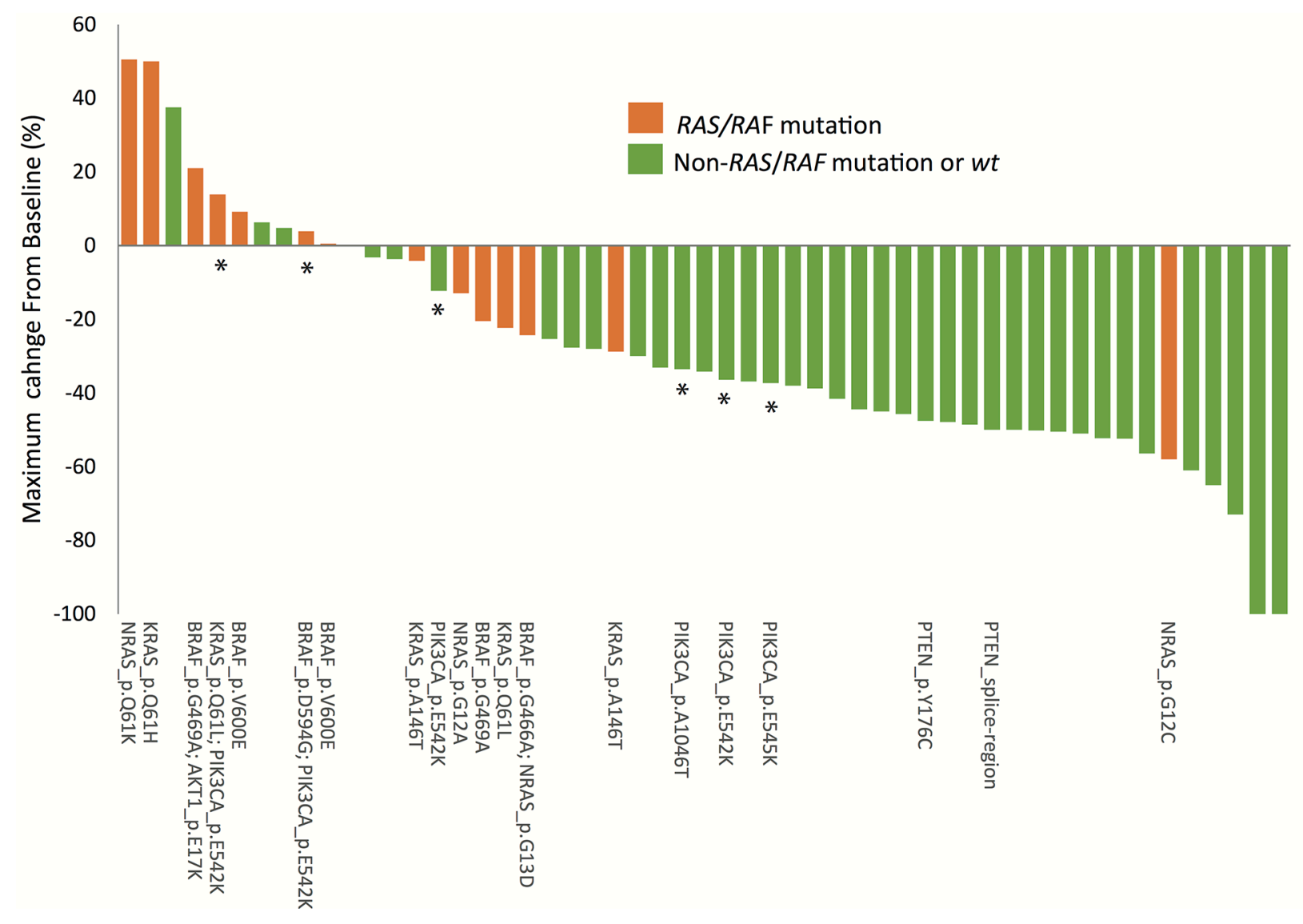

Figure 4: Relationship between responses to cetuximab-based treatment and genetic mutations. The waterfall plot depicts the percentage change in tumor size from baseline 3 months after cetuximab-based treatment. * indicates samples with mutations in PIK3CA. 
specimens. Applying the same approach may provide companion diagnostic tools for screening many other cancer-related genes in a multiplex, high throughput format.

The BRAF V600E substitution is a wellcharacterized oncogenic mutation in cancers such as melanoma. Previous studies found that $B R A F$ mutations are confined to codon 600 in CRC tissues. In this study, we identified three additional mutations on exons 11 and 15 of the $B R A F$ gene. Importantly, V600E mutations accounted for only one third of all $B R A F$ substitutions in the Taiwanese cohort. In addition, $11 \%$ of $\mathrm{mCRC}$ patients in this cohort carried $B R A F$ mutations, which is a larger proportion than observed previously in Caucasian populations. Our data illustrate that $B R A F$ mutation types and frequencies may vary depending on the ethnic background, gender, and disease severity of patients. $B R A F$ is strongly associated with metastasis, and $B R A F$ mutations along with increased kinase activity usually upregulate MAPK cascade signaling [24]. Our results unequivocally indicate that various $B R A F$ mutations were associated with shorter PFS in non-responders, suggesting that $B R A F$ mutations may help identify those who would benefit from anti-EGFR treatment among Taiwanese patients with wild-type $K R A S$. Although the predictive value of $B R A F$ mutations has been controversial in targeted therapies for mCRC [25-27], our study and many others demonstrate the utility of $B R A F$. For example, a meta-analysis by Wang et al. suggested that genetic aberrations in $B R A F$ impair the efficacy of anti-EGFR therapy in Asian mCRC populations (4). A recent study by Nicolantonio et al. also found that $B R A F$ mutations were associated with unfavorable clinical outcomes for cetuximab- or panitumumab-based therapies [28]. Further cell-based functional studies would clarify the significance of each $B R A F$ mutant allele that contributes to CRC carcinogenesis.

PIK3CA kinase and PTEN phosphatase are potent regulators in the PI3K-AKT-mTOR pathway, and mutations in these genes have long been implicated in various tumor types, including breast, colorectal, endometrial, ovary, liver, and gastric malignancies, despite low incidences [29]. Nevertheless, there are conflicting reports regarding the association between $P I K 3 C A$ mutations and resistance to cetuximab and panitumumab therapies in CRC [30-31]. Our results suggest that mutations in the PIK3CA-PTEN-AKT branch of the EGFR pathway have a lesser impact on malignant progression in
A

C

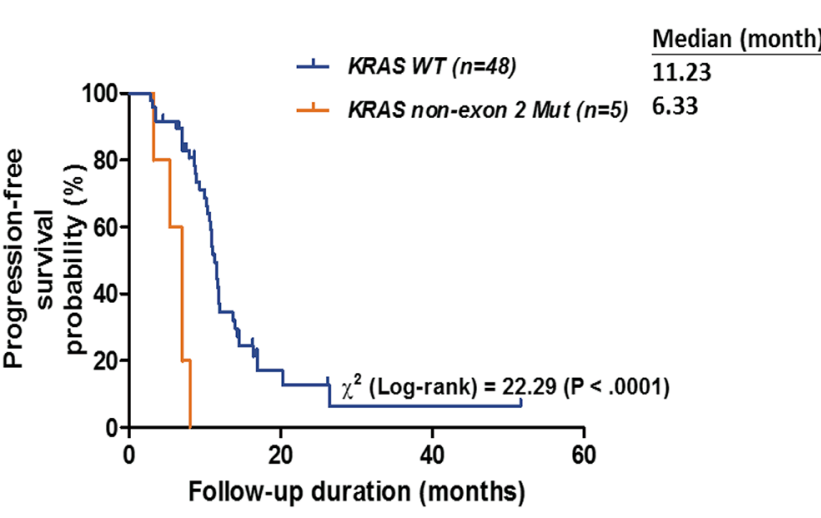

B

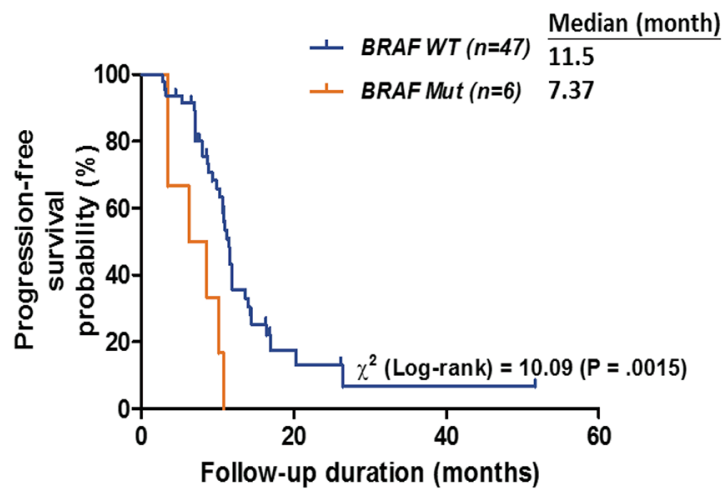

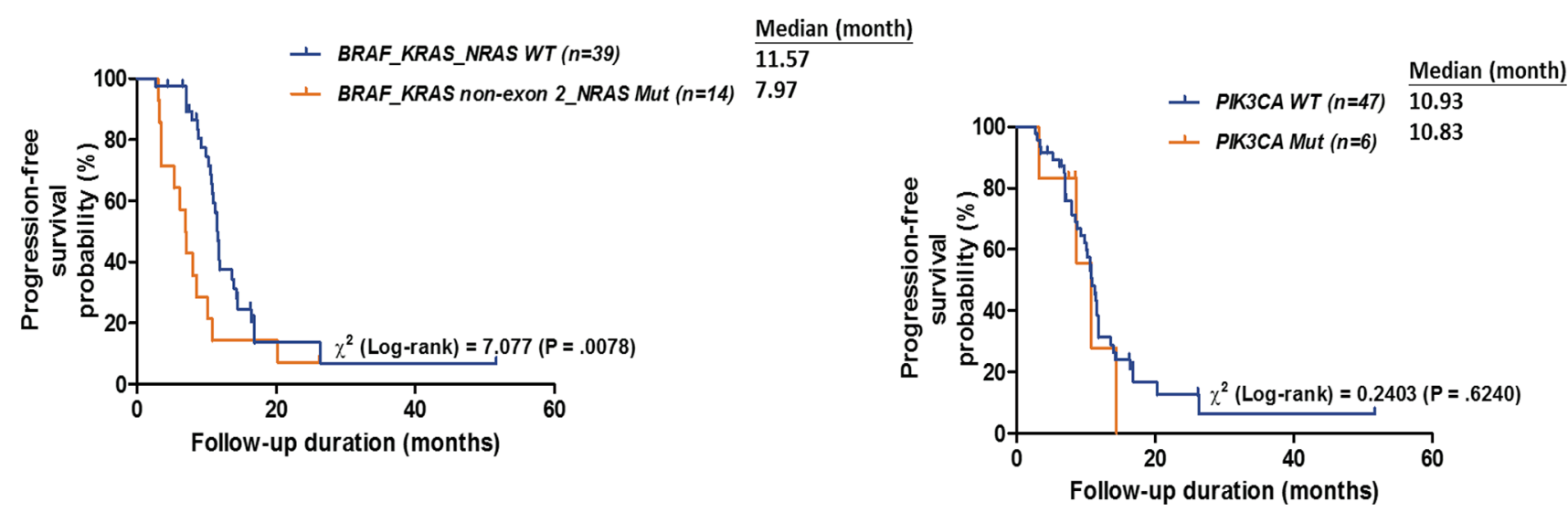

Figure 5: Kaplan-Meier plots for DSS according to the mutation status of KRAS (A), BRAF (B), BRAF, KRAS or NRAS (C) and PIK3CA (D). $p$ values were calculated using the log-rank test. 
Table 3: Univariate and multivariate analysis of factors associated with progression-free survival

\begin{tabular}{|c|c|c|c|c|c|c|c|}
\hline \multirow[b]{2}{*}{ Factors } & \multirow{2}{*}{$\frac{n=53}{\text { No. }}$} & \multicolumn{3}{|c|}{ Univariate analysis } & \multicolumn{3}{|c|}{ Multivariate analysis } \\
\hline & & HR & $95 \% \mathrm{CI}$ & $p$-value & HR & $95 \% \mathrm{CI}$ & $p$-value \\
\hline \multicolumn{8}{|l|}{ Clinical features } \\
\hline Sex (Male/Female) & $34 / 19$ & 0.911 & $0.483-1.720$ & 0.775 & & & \\
\hline Age $(>70 / \leq 70)$ & $13 / 40$ & 2.113 & $1.079-4.138$ & 0.029 & 1.926 & $0.964-3.846$ & 0.063 \\
\hline pT stage (T4/T23) & $22 / 31$ & 1.621 & $0.870-3.017$ & 0.128 & & & \\
\hline pN stage (N2/N01) & $28 / 25$ & 1.45 & $0.781-2.692$ & 0.239 & & & \\
\hline $\begin{array}{l}\text { Stage (stage4/ } \\
\text { stage1-3) }\end{array}$ & $35 / 18$ & 0.74 & $0.387-1.415$ & 0.363 & & & \\
\hline $\begin{array}{l}\text { Histologic Grade } \\
\text { (high/low) }\end{array}$ & $6 / 47$ & 2.055 & $0.855-4.939$ & 0.108 & & & \\
\hline $\begin{array}{l}\text { Metastatic pattern } \\
\text { (meta/syn) }\end{array}$ & $17 / 36$ & 1.247 & $0.646-2.406$ & 0.510 & & & \\
\hline $\begin{array}{l}\text { Primary Tumor site } \\
\text { (rectum/colon) }\end{array}$ & $21 / 32$ & 0.936 & $0.504-1.737$ & 0.834 & & & \\
\hline $\begin{array}{l}\text { Number of metastatic } \\
\text { sites }(>1 / 1)\end{array}$ & $22 / 31$ & 3.625 & $1.847-7.112$ & $<0.001$ & 3.668 & $1.828-7.360$ & $<0.001$ \\
\hline $\begin{array}{l}\text { Treatment regimen } \\
\text { (3rd line/1st line) }\end{array}$ & $14 / 39$ & 0.725 & $0.357-1.472$ & 0.373 & & & \\
\hline \multicolumn{8}{|l|}{ Genetic alteration } \\
\hline KRAS (Mut/WT) & $5 / 48$ & 9.502 & $3.056-29.55$ & $<0.001$ & 8.479 & $2.524-28.49$ & 0.001 \\
\hline BRAF (Mut/WT) & $6 / 47$ & 4.009 & $1.583-10.15$ & 0.003 & 2.603 & $1.017-6.661$ & 0.046 \\
\hline NRAS (Mut/WT) & $4 / 49$ & 0.656 & $0.193-2.227$ & 0.499 & & & \\
\hline $\begin{array}{l}\text { KRAS/NRAS } \\
\text { (Mut/WT) }\end{array}$ & $9 / 44$ & 1.779 & $0.795-3.982$ & 0.161 & & & \\
\hline $\begin{array}{l}\text { BRAF/KRAS/NRAS } \\
\text { (Mut/WT) }\end{array}$ & $14 / 39$ & 2.447 & $1.239-4.832$ & 0.010 & 2.716 & $1.345-5.481$ & 0.005 \\
\hline
\end{tabular}

CRC than mutations in the RAS-RAF-MAPK branch. It is also noteworthy that the majority of PIK3CA mutations in this study were found in exon 9 , while previous studies have suggested that $P I K 3 C A$ exon 20 , but not $P I K 3 C A$ exon 9 , mutations are associated with cetuximab resistance in CRC [22]. Although the association between PIK3CA mutations and treatment resistance was not statistically significant, individual PIK3CA mutations tended to be associated with poorer clinical outcomes as indicated by increased SD or marginal PR. In contrast, tumors with concomitant mutations in PIK3CA and KRAS or in $P I K 3 C A$ and $B R A F$ were more resistant to cetuximab therapy as indicated by increased PD.

PTEN mutations are found in normal and neoplastic cells, and the significance of PTEN mutations in carcinogenesis is also unclear [32]. Several immunohistochemistry-based studies have linked loss of PTEN expression to the anti-tumor activity of cetuximab in advanced CRC [33-34]. However, we detected PTEN mutations in a small number of responders, suggesting either that PTEN mutations are poor predictors of response to cetuximab or that different experimental methods are needed when using PTEN as a biomarker in clinical routine diagnosis. The PTEN Y176C mutation only modestly decreased its catalytic activity and conformational stability [35], which could explain why carriers of this mutation still benefit from anti-EGFR therapy. The AKTE17K mutation has been linked to prolonged activation of the gene, which contributes to tumorigenesis by over-activating the mTOR pathway [36]. However, because the one non-responsive subject with an $A K T$ mutation also carried a $B R A F$ mutation, it was difficult for us to examine the individual predictive value of $A K T$ for anti-EGFR treatment. Taken together, although our data indicate that mutations in PIK3CA, PTEN and AKT do not predict anti-EGFR therapy efficacy, more studies are needed to further evaluate these genes' clinical significance. 
Because no mutations in any of the genes examined here were detected in 10 non-responsive subjects, it is likely that other carcinogenesis-associated genes also influence resistance to cetuximab treatment. Other factors, including increases in $E G F R$ or $A L K$ gene copy numbers, $M E T$ overexpression, and elevated KRAS, MET, and ERBB levels following cetuximab therapy, have been linked to CRC progression and deserve further investigation [37-39]. Finally, only surgically removed pre-treatment primary tumor tissues were analyzed in the current study. Previous studies have found some differences in $K R A S$ mutation status between primary tumors and corresponding metastatic lesions [40-41]. Such genetic heterogeneity may explain why no additional mutations were detected in the non-responders.

In conclusion, we provide evidence that the detection of EGFR-pathway variants aids in the identification of EGFR therapy non-responders. Our data strongly suggest that mutation screening should be extended beyond $K R A S$ exon 2 to include $K R A S$ exons 3 and 4, BRAF, and NRAS. These variants were associated with poor tumor responses and shorter PFS in mCRC patients with wild-type KRAS who were treated with the anti-EGFR agent cetuximab.

\section{METHODS}

\section{Patients}

Fifty-three consecutive patients with $\mathrm{mCRC}$ who were treated between 2010 and 2014 at the Oncology Department of the Chang Gung Memorial Hospital, TaoYuan, Taiwan, were included in this study (see Table 1 for an overview of patient characteristics). Inclusion criteria were: (1) histologically confirmed colorectal adenocarcinoma, (2) metachronous or synchronous metastatic disease, (3) wild-type $K R A S$ exon 2 in primary colorectal or metastatic tumors (confirmed by direct sequencing performed in the Pathology Department at Chang Gung, detection limit: $20 \%$ ), (iv) previous combined cetuximab and oxaliplatinor irinotecan-based chemotherapy treatment, (v) measurable radiological lesions, and (vi) good performance status (ECOG: 0-1). The study was approved by the Institutional Review Board at the Chang Gung Memorial Hospital (IRB 102-2850A3). Written informed consent was obtained from all patients before sample collection.

\section{Treatment regimens}

In the first-line therapy group, patients with mCRC received cetuximab plus chemotherapy with FOLFIRI (irinotecan and infusional 5-fluorouracil with leucovorin) [8]. In the third-line group, patients with $\mathrm{mCRC}$ who experienced progression after irinotecan and oxaliplatin-based chemotherapy received cetuximab plus chemotherapy with IFL (irinotecan and bolus 5 -fluorouracil with leucovorin) [42-43]. The detailed chemotherapy and cetuximab schedules were as follows: (i) IFL (Leucovorin $20 \mathrm{mg} / \mathrm{m}^{2}$ iv bolus qw x 4 weeks every 6 weeks, 5 -FU $500 \mathrm{mg} / \mathrm{m}^{2}$ iv bolus qw x 4 weeks every 6 weeks, irinotecan $125 \mathrm{mg} / \mathrm{m}^{2}$ iv qw x 4 weeks every 6 weeks for 4 cycles) [42]; (ii) FOLFIRI regimen (Leucovorin $400 \mathrm{mg} / \mathrm{m}^{2}$ iv over 2 hours before 5 -FU d1, 5 -FU $400 \mathrm{mg} / \mathrm{m}^{2}$ iv bolus $\mathrm{d} 1$, and then $2400 \mathrm{mg} / \mathrm{m}^{2}$ iv over 46 hours, irinotecan $180 \mathrm{mg} /$ $\mathrm{m}^{2}$ iv over 90 min d 1 every 2 weeks for 12 cycles) [45]. (iii). Cetuximab infusion (with a loading dose of $400 \mathrm{mg} /$ $\mathrm{m}^{2}$ and subsequent maintenance doses of $250 \mathrm{mg} / \mathrm{m}^{2} /$ per week or $500 \mathrm{mg} / \mathrm{m}^{2}$ biweekly) [42-44, 46-47]. Toxicity was evaluated using Common Terminology Criteria (CTCAE) for Adverse Events, version 4.0 [48].

\section{Sample preparation, DNA sequencing, and data processing}

Surgically removed pre-treatment primary tumor tissue specimens were fixed in formalin and preserved in paraffin blocks for histological examination and for prolonged storage. Two $10 \mu \mathrm{m}$-thick formalin-fixed paraffin-embedded (FFPE) sections were used for this study. Genomic DNA was extracted from FFPE tumor samples using the QIAamp DNA FFPE Tissue Kit (Qiagen). DNA was quantified using the Quant-iT dsDNA HS Assay (Invitrogen). The integrity of genomic DNA was determined using the Fragment Analyzer assay (Advanced Analytical Technologies, Inc.) and quantitative PCR.

Twenty ng of genomic DNA were amplified using a pool of primers to enrich frequently mutated hotspot regions of 10 genes that are related to the EGFR pathway (EGFR, KRAS, HRAS, NRAS, BRAF, PIK3CA, AKT1, PTEN, HER2, and HER4) and TP53. Complete targeted regions and primer sequences are listed in supplementary Table 2. The entire sequencing region covered 6225 bases and included 2611 entries from the Catalogue of Somatic Mutation in Cancer (COSMIC) database (version 68). Amplicons were ligated with barcoded adaptors using the Ion Amplicon Library Kit (Life Technologies). Barcoded libraries were subsequently conjugated with sequencing beads by emulsion PCR and enriched using Ion OneTouch2 and OneTouch ES (Life Technologies) according to the manufacturer's protocol. Sequencing was performed using the Ion Torrent PGM system with the Ion 318 chip. Raw reads were mapped to the hg19 reference genome using Torrent Suite Server (version 4.2) and variants were identified using the Torrent Variant Caller plug-in (version 4.2). Per the manufacturer's recommendation, the variant calling threshold was set at $5 \%$ for variants without COSMIC IDs and $2 \%$ for variants with COSMIC IDs. Variants were annotated using the Variant Effect Predictor (VEP, version 78). Common variants (MAF $\geq 1 \%$ ) in the dbSNP database (build 138) or in the 1000 Genome project (phase 1) without COSMIC entries were filtered out. Only non-synonymous mutations were analyzed. 


\section{Statistical analysis}

Efficacy of anti-EGFR therapy was evaluated by a CT scan every 3 months according to RECIST criteria version 1.1 [49]. Patients were categorized as responders if they achieved complete response (CR) or partial response (PR) and non-responders if they demonstrated stable disease (SD) or progressive disease (PD). Progression-free survival (PFS) was calculated from the first day of therapy to the date of proven progression, or death. The last date when the patient was seen alive without recurrence was used for those who could not be located for follow-up.

The significance of the association between individual clinical factors was evaluated using Fisher's exact test. The survival rate was calculated by the Kaplan-Meier method and the statistical significance of the differences was evaluated using the log-rank test. All statistical tests were two-sided, and $p<0.05$ was considered statistically significant. All statistical analyses were performed using SPSS version 18.0 for Windows (SPSS Inc., Chicago, IL., USA).

\section{CONFLICTS OF INTEREST}

Tan Kien Thiam, Yen-Jung Lu, An Hsu, Hua-Chien Chen and Shu-Jen Chen are employees of ACT Genomics.

\section{GRANT SUPPORT}

This work was supported by grants from Chang Gung Memorial Hospital, Taiwan (CMRPG3C1841) to H.C. Hsu.

\section{REFERENCES}

1. Parkin DM, Bray F, Ferlay J, Pisani P. Global cancer statistics. 2002. CA Cancer J Clin. 2005; 55:74-108.

2. Wieser M, Sauerland S, Arnold D, Schmiegel W, ReinacherSchick A. Peri-operative chemotherapy for the treatment of resectable liver metastases from colorectal cancer: a systematic review and metaanalysis of randomized trials. BMC Cancer. 2010; 10:309.

3. Vermaat JS, Nijman IJ, Koudijs MJ, Gerritse FL, Scherer SJ, Mokry M, Roessingh WM, Lansu N, de Bruijn E, van Hillegersberg R, van Diest PJ, Cuppen E, Voest EE. Primary colorectal cancers and their subsequent hepatic metastases are genetically different: implications for selection of patients for targeted treatment. Clin Cancer Res. 2012; 18:688-990.

4. Lee SY, Haq F, Kim D, Jun C, Jo HJ, Ahn SM, Lee WS. Comparative genomic analysis of primary and synchronous metastatic colorectal, cancers. PLoS ONE. 2014; 9: e90459.
5. Cao Y. VEGF-targeted cancer therapeutics-paradoxical effects in endocrine organs. Nat Rev Endocrinol. 2014; 10:530-9.

6. Ciardiello FT, ortora GE. GFR antagonists in cancer treatment. N Engl J Med. 2008; 358:1160-74.

7. Van Cutsem E, Kohne CH, Lang I, Folprecht G, Nowacki MP, Cascinu S, Shchepotin I, Maurel J, Cunningham D, Tejpar S, Schlichting M, Zubel A, Celik I, et al. Cetuximab plus irinotecan, fluorouracil, and leucovorin as firstline treatment for metastatic colorectal cancer: updated analysis of overall survival according to tumor KRAS and BRAF mutation status. J Clin Oncol Off J Am Soc Clin Oncol. 2011; 29:2011-2019.

8. Van Cutsem E, Kohne CH, Hitre E, Zaluski J, Chang Chien CR, Makhson A, D’Haens G, Pintér T, Lim R, Bodoky G, Roh JK, Folprecht G, Ruff P, et al. Cetuximab and chemotherapy as initial treatment for metastatic colorectal cancer. N Eng J Med. 2009; 360:1408-1417.

9. Saltz LB, Meropol NJ, Loehrer PJ Sr Needle MN, Kopit J, Mayer RJ. Phase II trial of cetuximab in patients with refractory colorectal cancer that expresses the epidermal growth factor receptor. J Clin Oncol. 2004; 22:1201-8.

10. Van Cutsem E, Peeters M, Siena S, Humblet Y, Hendlisz A, Neyns B, Canon JL, Van Laethem JL, Maurel J, Richardson G, Wolf M, Amado RG, et al. Open-label phase III trial of panitumumab plus best supportive care compared with best supportive care alone in patients with chemotherapyrefractory metastatic colorectal cancer. J Clin Oncol. 2007; 25:1658-64.

11. Bokemeyer C, Bondarenko I, Makhson A, Hartmann JT, Aparicio J, de Braud F, Donea S, Ludwig H, Schuch G, Stroh C, Loos AH, Zubel A, Koralewski P, et al. Fluorouracil, leucovorin, and oxaliplatin with and without cetuximab in the fi rst-line treatment of metastatic colorectal cancer. J Clin Oncol. 2009; 27:663-71.

12. Therkildsen C, Bergmann TK, Henrichsen-Schnack T, Ladelund S, Nilbert M. The predictive value of KRAS, NRAS, BRAF, PIK3CA and PTEN for anti-EGFR treatment in metastatic colorectal cancer: A systematic review and meta-analysis. Acta Oncol. 2014; 53:852-64.

13. Cheng L, Ren W, Xie L, Li M, Liu J, Hu J, Liu BR, Qian XP. Anti-EGFR MoAb treatment in colorectal cancer: limitations, controversies, and contradictories. Cancer Chemother Pharmacol. 2014; 74:1-13.

14. El-Telbany A, Ma PC. Cancer genes in lung cancer: racial disparities: arethere any? Genes Cancer. 2012; 3:467-80. doi: 10.1177/1947601912465177.

15. Voelkerding KV, Dames SA, Durtschi JD. Next-generation sequencing: from basic research to diagnostics. Clin Chem. 2009; 55:641-58.

16. Brannon AR, Vakiani E, Sylvester BE, Scott SN, McDermott G, Shah RH, Kania K, Viale A, Oschwald DM, Vacic V, Emde AK, Cercek A, Yaeger R, et al. Comparative 
sequencing analysis reveals high genomic concordance between matched primary and metastatic colorectal cancer lesions. Genome biology. 2014; 15:454

17. Cancer Genome Atlas Network. Comprehensive molecular characterization of human colon and rectal cancer. Nature. 2012; 487:330-7.

18. Imamura Y, Lochhead $P$, Yamauchi M, Kuchiba A, Qian ZR, Liao X, Nishihara R, Jung S, Wu K, Nosho K, Wang YE, Peng S, Bass AJ, et al. Analyses of clinicopathological, molecular, and prognostic associations of KRAS codon. 61 and codon. 146 mutations in colorectal cancer: cohort study and literature review. Mol Cancer. 2014; 13:135.

19. Takahashi N, Yamada Y, Taniguchi H, Honma Y, Iwasa S, Kato K, Hamaguchi T, Shimada Y. Combined assessment of epidermal [corrected] growth factor receptor dual color in situ hybridization and immunohistochemistry with downstream gene mutations in prediction of response to the anti-EGFR therapy for patients withmetastatic colorectal cancer. Arch Med Res. 2014; 45:366-74.

20. Bekaii-Saab T. Moving forward with expanding to an "allRAS mutational analysis" in metastatic colorectal cancer: beyond KRAS mutations. J Natl Compr Canc Netw. 2014; 12:299-300.

21. Saridaki Z, Tzardi M, Sfakianaki M, Papadaki C, Voutsina A, Kalykaki A, Messaritakis I, Mpananis K, Mavroudis D, Stathopoulos E, Georgoulias V'1 , Souglakos J. BRAFV600E mutation analysis in patients with metastatic colorectal cancer (mCRC) in daily clinical practice: correlations with clinical characteristics, and its impact on patients' outcome. PLoS one. 2013; 8, e84604.

22. De Roock W, Claes B, Bernasconi D, De Schutter J, Biesmans B, Fountzilas G, Kalogeras KT, Kotoula V, Papamichael D, Laurent-Puig P, Penault-Llorca F, Rougier P, Vincenzi B, et al. Effects of KRAS, BRAF, NRAS, and PIK3CA mutations on the efficacy of cetuximab plus chemotherapy in chemotherapy-refractory metastatic colorectal cancer: a retrospective consortium analysis. The Lancet. Oncology. 2010; 11:753-762

23. Soeda H, Shimodaira H, Watanabe M, Suzuki T, Gamoh M, Mori T, Komine K, Iwama N, Kato S, Ishioka C. Clinical usefulness of KRAS, BRAF, and PIK3CA mutations as predictive markers of cetuximab efficacy in irinotecan- and oxaliplatin-refractory Japanese patients with metastatic colorectal cancer. International journal of clinical oncology. 2013; 18:670-677.

24. Fearon ER. Molecular genetics of colorectal cancer. Annual review of pathology. 2011; 6:479-507.

25. Roth AD, Tejpar S, Delorenzi M, Yan P, Fiocca R, KlingbielD, Dietrich D, Biesmans B, Bodoky G, Barone C. Aranda E, Nordlinger B, Cisar L, et al. Prognostic role of KRAS and BRAF in stage II and III resected colon cancer: results of the translational study on the PETACC-3, EORTC 40993, SAKK 60-00 trial. J Clin Oncol. 28:466-474.
26. Tran B, Kopetz S, Tie J, Gibbs P, Jiang ZQ, Lieu CH, Agarwal A, Maru DM, Sieber O, Desai J. Impact of BRAF mutation and microsatellite instability on the pattern of metastatic spread and prognosis in metastatic colorectal cancer. Cancer; 2011; 117:4623-4632.

27. Wang Q, Hu WG, Song QB, Wei J. BRAF V600E Mutation as a Predictive Factor of Anti-EGFR Monoclonal Antibodies Therapeutic Effects in Metastatic Colorectal Cancer: a Meta-analysis. Chinese medical sciences journal. 2014; 29:197-203.

28. Di Nicolantonio F, Martini M, Molinari F, Sartore-Bianchi A, Arena S, Saletti P, De Dosso S, Mazzucchelli L, Frattini M, Siena S, Bardelli A. Wild-type BRAF is required for response to panitumumab or cetuximab in metastatic colorectal cancer. J Clin Oncol. 2008; 26:5705-5712.

29. Samuels Y, Wang Z, Bardelli A, Silliman N, Ptak J, Szabo S, Yan H, Gazdar A, Powell SM, Riggins GJ, Willson JK, Markowitz S, Kinzler KW, et al. High frequency of mutations of the PIK3CA gene in human cancers. Science. 2004; 304:554.

30. Prenen H, De Schutter J, Jacobs B, De Roock W, Biesmans B, Claes B, Lambrechts D, Van Cutsem E, Tejpar S. PIK3CA mutations are not a major determinant of resistance to the epidermal growth factor receptor inhibitor cetuximab in metastatic colorectal cancer. Clinical cancer research. 2009; 15:3184-3188.

31. Perrone F, Lampis A, Orsenigo M, Di Bartolomeo M, Gevorgyan A, Losa M, Frattini M, Riva C, Andreola S, Bajetta E, Bertario L, Leo E, Pierotti MA, et al. PI3KCA/ PTEN deregulation contributes to impaired responses to cetuximab in metastatic colorectal cancer patients. Annals of oncology. 2009; 20:84-90.

32. Sarquis MS, Agrawal S, Shen L, Pilarski R, Zhou XP, and Eng C. Distinct expression profiles for PTEN transcript and its splice variants in Cowden syndrome and BannayanRiley-Ruvalcaba syndrome. American journal of human genetics. 2006; 79:23-30.

33. Negri FV, Bozzetti C, Lagrasta CA, Crafa P, Bonasoni MP, Camisa R, Pedrazzi G, Ardizzoni A. PTEN status in advanced colorectal cancer treated with cetuximab. British journal of cancer. 2010; 102:162-164.

34. Loupakis F, Pollina L, Stasi I, Ruzzo A, Scartozzi M, Santini D, Masi G, Graziano F, Cremolini C, Rulli E. Canestrari E, Funel N, Schiavon G, et al. PTEN expression and KRAS mutations on primary tumors and metastases in the prediction of benefit from cetuximab plus irinotecan for patients with metastatic colorectal cancer. Journal of clinical oncology. 2009; 27:2622-2629.

35. Johnston SB, Raines RT. Conformational Stability and Catalytic Activity of PTEN Variants Linked to Cancers and Autism Spectrum Disorders. Biochemistry. 2015; 54: 1576-82.

36. Carpten JD, Faber AL, Horn C, Donoho GP, Briggs SL, Robbins CM, Hostetter G, Boguslawski S, Moses TY, 
Savage S. Uhlik M, Lin A, Du J, et al. A transforming mutation in the pleckstrin homology domain of AKT1 in cancer. Nature. 2007; 448:439-444

37. Yuan ZX, Wang XY, Qin QY, Chen DF, Zhong QH, Wang L, Wang JP. The prognostic role of BRAF mutation in metastatic colorectal cancer receiving anti-EGFR monoclonal antibodies: a meta-analysis. PLoS one. 2013; 8, e65995.

38. Kishiki T, Ohnishi H, Masaki T, Ohtsuka K, Ohkura Y, Furuse J, Watanabe T, Sugiyama M. Overexpression of MET is a new predictive marker for anti-EGFR therapy in metastatic colorectal cancer with wild-type KRAS. Cancer chemotherapy and pharmacology. 2014; 73:749-757.

39. Mohan S, Heitzer E, Ulz P, Lafer I, Lax S, Auer M, Pichler M, Gerger A, Eisner F, Hoefler G, Bauernhofer T, Geigl JB, Speicher MR. Changes in colorectal carcinoma genomes under anti-EGFR therapy identified by wholegenome plasma DNA sequencing. PLoS genetics. 2014; 10, e1004271.

40. Knijn N, Mekenkamp LJ, Klomp M, Vink-Börger ME, Tol J, Teerenstra S, Meijer JW, Tebar M, Riemersma S, van Krieken JH, Punt CJ, Nagtegaal ID. KRAS mutation analysis: a comparison between primary tumours and matched liver metastases in 305 colorectal cancer patients. Br J Cancer. 2011; 15; 104:1020-6.

41. Watanabe T, Kobunai T, Yamamoto Y, Matsuda K, Ishihara S, Nozawa K, Iinuma H, Shibuya H, Eshima K. Heterogeneity of KRAS status may explain the subset of discordant KRAS status between primary and metastatic colorectal cancer. Dis Colon Rectum. 2011; 54:1170-8.

42. Cunningham D, Humblet Y, Siena S, Khayat D, Bleiberg H, Santoro A, Bets D, Mueser M, Harstrick A, Verslype C, Chau I, Van Cutsem E. Cetuximab monotherapy and cetuximab plus irinotecan in irinotecan-refractory metastatic colorectal cancer. N Engl J Med. 2004; 351:337.
43. Sobrero AF, Maurel J, Fehrenbacher L, Scheithauer W, Abubakr YA, Lutz MP, Vega-Villegas ME, Eng C, Steinhauer EU, Prausova J, Lenz HJ, Borg C, Middleton G, et al. EPIC: phase III trial of cetuximab plus irinotecan after fluoropyrimidine and oxaliplatin failure in patients with metastatic colorectal cancer. J Clin Oncol. 2008; 26:2311.

44. Saltz LB, Cox JV, Blanke C, Rosen LS, Fehrenbacher L, Moore MJ, Maroun JA, Ackland SP, Locker PK, Pirotta N, Elfring GL, Miller LL. IFL Irinotecan plus fluorouracil and leucovorin for metastatic colorectal cancer. Irinotecan Study Group. N Engl J Med. 2000; 343:905-914.

45. Fuchs CS, Marshall J, Mitchell E, Wierzbicki R, Ganju V, Jeffery M, Schulz J, Richards D, Soufi-Mahjoubi R, Wang B, Barrueco J. Randomized, controlled trial of irinotecan plus infusional, bolus, or oral fluoropyrimidines in first-line treatment of metastatic colorectal cancer: results from the BICC-C Study. J Clin Oncol. 2007; 25:4779-4786.

46. Hubbard JM, Alberts SR. Alternate dosing of cetuximab for patients with metastatic colorectal cancer. Gastrointest Cancer Res. 2013; 6:47-55.

47. Chen Y, Cao D, Bi F, Li Q, Qiu M. Biweekly cetuximab plus FOLFIRI /irinotecan as first/second-line chemotherapy for patients with KRAS wild-type metastatic colorectal cancer: a retrospective analysis in Southwest Chinese population. Med Oncol. 2014; 31:935.

48. NCI. CTCAE. Available from: URL:http://evs.nci.nih.gov/ ftp1/ CTCAE /About.html. Accessed May. 17:2010.

49. Eisenhauer EA, Therasse P, Bogaerts J, Schwartz LH, Sargent D, Ford R, Dancey J, Arbuck S, Gwyther S, Mooney M, Rubinstein L, Shankar L, Dodd L, et al. New response evaluation criteria in solid tumours: revised RECIST guideline (version 1.1). Eur J Cancer. 2009; 45:228-47. 